\title{
Josephson junctions in a local inhomogeneous magnetic field
}

\author{
V. M. Krasnov ๑* \\ Department of Physics, Stockholm University, AlbaNova University Center, SE-10691 Stockholm, Sweden \\ and Moscow Institute of Physics and Technology, State University, 9 Institutsiy per., Dolgoprudny, Moscow Region 141700 Russia
}

(Received 4 February 2020; accepted 3 April 2020; published 23 April 2020)

\begin{abstract}
A Josephson junction can be subjected to a local, strongly inhomogeneous magnetic field in various experimental situations. Here this problem is analyzed analytically and numerically. A modified sine-Gordon type equation in the presence of time-dependent local field is derived and solved numerically in static and dynamic cases. Two specific examples of local fields are considered: induced either by an Abrikosov vortex, or by a tip of a magnetic force microscope (MFM). It is demonstrated that a time-dependent local field can induce a dynamic flux-flow state in the junction with shuttling or unidirectional ratchetlike Josephson vortex motion. This provides a mechanism for detection and manipulation of Josephson vortices by an oscillating MFM tip. In a static case, the local field leads to a distortion of the critical current versus magnetic field, $I_{c}(H)$, pattern. The distortion is sensitive to both the shape and the amplitude of the local field. Therefore, the $I_{c}(H)$ pattern carries information about the local field distribution within the junction. This opens the possibility for employing a single Josephson junction as a scanning probe sensor with spatial resolution not limited by its geometrical size, thus obviating a known problem of a trade-off between the field sensitivity and the spatial resolution of a sensor.
\end{abstract}

DOI: 10.1103/PhysRevB.101.144507

\section{INTRODUCTION}

Properties of Josephson junctions (JJs) with spatially uniform parameters in a homogenous magnetic field are well studied [1]. Spatially nonuniform JJs in a homogeneous field were also considered earlier [2-9]. However, in many experimental situations, JJs are subjected to a local, strongly inhomogeneous magnetic field. For example, it can originate from a self-induced flux in JJs with a sign-reversal order parameter [10-12]; appear in JJs containing ferromagnetic interlayers with spatially inhomogeneous thicknesses [13-16], nanoparticles, or domain walls [17-22]; in JJs with a local current injection $[23,24]$; and can be induced by a nearby Abrikosov vortex [25-28], by a sharp tip of a magnetic force microscope (MFM) [29,30], etc. Although such a situation has been considered previously, often this has been done without proper substantiation. To my knowledge, there is no established formalism for a general treatment of such a problem, especially in the dynamic case.

Another motivation of this paper is related to a recent proposal to use a single planar JJ as a scanning probe sensor [31]. The leading superconducting scanning probe technique today is the scanning SQUID (superconducting quantum interference device) microscopy $[32,33]$. Despite many advantages, SQUIDs suffer from a trade-off problem between the field

\footnotetext{
*Vladimir.Krasnov@fysik.su.se
}

Published by the American Physical Society under the terms of the Creative Commons Attribution 4.0 International license. Further distribution of this work must maintain attribution to the author(s) and the published article's title, journal citation, and DOI. Funded by Bibsam. sensitivity and the spatial resolution. SQUIDs, as well as most other superconducting magnetic sensors, are measuring flux with a resolution $\delta \Phi$ determined by the flux quantum $\Phi_{0}$. Therefore, the field sensitivity is inversely proportional to the sensor (pickup loop) area $S, \delta H=\delta \Phi / S$. On the other hand, the spatial resolution is determined by the sensor size $\delta x \sim$ $S^{1 / 2}$. Consequently, the better the spatial resolution, the worse the field sensitivity, $\delta H \sim 1 / \delta x^{2}$. In Ref. [31], it was argued that a sensor based on a single planar JJ would be able to obviate this trade-off problem, at least in one spatial direction. Similar to a SQUID, the field sensitivity of a planar JJ is also inversely proportional to the area. Therefore, obviation of the trade-off problem would require independence of the spatial resolution on the junction size. That is, the junction should be able to resolve a spatial variation of magnetic field at a scale significantly smaller than the junction length. This brings us again to the problem of a JJ in a local spatially inhomogeneous magnetic field.

In this paper, I consider analytically and numerically a response of a single $\mathrm{JJ}$ to a local inhomogeneous and timedependent magnetic field. First, a modified sine-Gordon equation for this case is derived. The equation is then solved numerically both for short and long junctions, and both in static and dynamic cases. Two specific examples (without losing generality) are considered with a local field induced either by an Abrikosov vortex or by a tip of MFM. It is demonstrated that a time-dependent local field can induce a dynamic flux-flow phenomenon with either shuttling or unidirectional ratchetlike motion of Josephson vortices $(\mathrm{JVs})$ in the junction, which provides a mechanism for detection of JVs by MFM [30]. Analysis of the static case shows how the critical current versus magnetic field, $I_{c}(H)$, modulation is distorted by the local field. Importantly, the shape of distorted $I_{c}(H)$ patterns depends both on the shape, amplitude, and position 
of the local field. Therefore, the $I_{c}(H)$ pattern carries detailed information about the local field and it should be possible to extract field distribution within the junction using proper mathematical treatment. This would open the possibility for making a scanning probe sensor based on a single planar JJ with spatial resolution not limited by its geometrical size, thus obviating the trade-off problem between sensitivity and resolution.

\section{THEORETICAL ANALYSIS}

Figure 1 represents a sketch of the studied problem. Let us consider a $\mathrm{JJ}$ in an applied uniform magnetic field $H$ (in the $y$ direction) and a local nonuniform field $B^{*}$ (generally with all three $x, y, z$ components). The bias current with density $J_{b}$ is applied from one electrode to another in the $z$ direction. The actual magnetic induction in the junction, $B \neq H$, is influenced by circulating screening currents in the junction, the local field $B^{*}$, and the field induced by the bias current, $B_{b}$. I start with derivation of transport equations. Although main parts of the derivation are well known, I will show it in some detail both for the sake of pedagogical completeness and because sometimes different equations have been introduced in literature without proper substantiation.

According to the two-fluid model, a current through JJs has superconducting and quasiparticle (normal) components. The supercurrent density is given by the DC-Josephson relation,

$$
J_{s}=J_{c 0} \sin (\varphi) \text {, }
$$

where $J_{c 0}$ is the critical current density at zero magnetic field and $\varphi$ is the Josephson phase difference. The quasiparticle current density is equal to $J_{n}=V / R_{n} A$, where $V$ is voltage across the junction, $R_{n}$ is the normal resistance of the junction, and $A$ is the junction area. Using the AC-Josephson relation $V=\left(\Phi_{0} / 2 \pi c\right)(\partial \varphi / \partial t)$, where $c$ is the speed of light in vacuum, it can be written as

$$
J_{n}=\frac{\Phi_{0}}{2 \pi c R_{n} A} \frac{\partial \varphi}{\partial t} .
$$

Note that for planar JJs the junction area $A$ in the $(x, y)$ plane, see Fig. 1, is much smaller than the sensor area $S$ in the $(x, z)$ plane [31,34]. Using Maxwell equation, $\operatorname{rot} B=\frac{4 \pi}{c} J+\frac{1}{c} \frac{\partial D}{\partial t}$, where $D$ is the displacement field, we can write the total

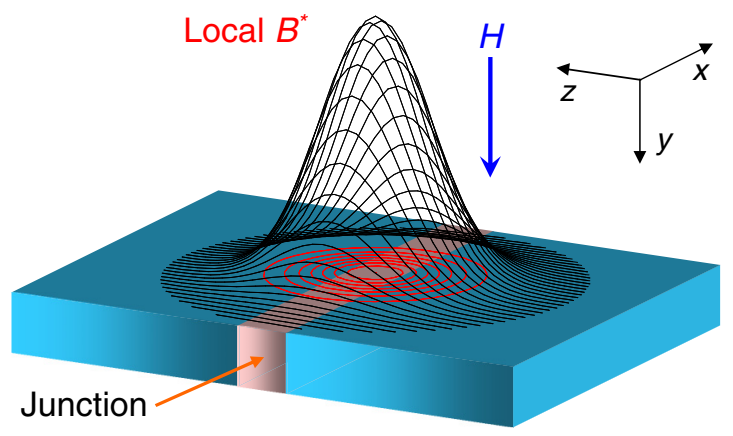

FIG. 1. Sketch of a Josephson junction in a local inhomogeneous magnetic field $B^{*}$. current through the junction as

$$
J_{z}=\frac{c}{4 \pi}\left(\frac{\partial B_{y}}{\partial x}-\frac{\partial B_{x}}{\partial y}\right)-\frac{1}{4 \pi} \frac{\partial D}{\partial t} .
$$

Here the last term represents the displacement current density,

$$
J_{D}=\frac{\Phi_{0} C}{2 \pi c A} \frac{\partial^{2} \varphi}{\partial t^{2}},
$$

where $C$ is the junction capacitance.

Both $x$ and $y$ components of the magnetic induction in Eq. (3) are essential. The $x$ component, parallel to the junction, has contributions from the bias current, $B_{x b}$, and the local nonuniform field $B_{x}^{*}, B_{x}=B_{x b}+B_{x}^{*}$. According to the Maxwell equation, the bias contribution is [35]

$$
\frac{\partial B_{x b}}{\partial y} \simeq-\frac{4 \pi}{c} J_{b}
$$

This is how the bias current, which plays the role of the driving force, enters the sine-Gordon equation. From Eqs. (1)-(5), we obtain

$$
J_{D}+J_{n}+J_{s}=\frac{c}{4 \pi}\left(\frac{\partial B_{y}}{\partial x}-\frac{\partial B_{x}^{*}}{\partial y}\right)+J_{b} .
$$

The $y$ component of magnetic field, going through the junction, induces a phase gradient in the junction:

$$
\frac{\partial \varphi}{\partial x}=\frac{2 \pi d_{\mathrm{eff}}}{\Phi_{0}} B_{y} .
$$

Here $d_{\text {eff }}$ is the so-called magnetic thickness of the junction. $B_{y}$ is the total (screened) induction in the junction subjected to the applied uniform field $H$, the local nonuniform field $B^{*}$, as well as the self-field $B_{y b}$ in the case of nonuniform bias [5,35]. $B_{y}$ is generally not known and should be determined. To do so, we separate the phase shift $\varphi^{*}$ caused solely by $B_{y}^{*}$ :

$$
\begin{gathered}
\varphi=\phi+\varphi^{*}, \\
\frac{\partial \varphi^{*}}{\partial x}=\frac{2 \pi d_{\mathrm{eff}}}{\Phi_{0}} B_{y}^{*}, \\
\frac{\partial \phi}{\partial x}=\frac{2 \pi d_{\mathrm{eff}}}{\Phi_{0}}\left(B_{y}-B_{y}^{*}\right) .
\end{gathered}
$$

$\varphi^{*}(x)$ is a known function, determined by integration of Eq. (9) along the junction length (up to an integration constant).

Using Eqs. (7)-(10), we can write

$$
J_{D}+J_{n}+J_{s}=\frac{c \Phi_{0}}{8 \pi^{2} d_{\mathrm{eff}}} \frac{\partial^{2} \phi}{\partial x^{2}}+\frac{c}{4 \pi}\left(\frac{\partial B_{y}^{*}}{\partial x}-\frac{\partial B_{x}^{*}}{\partial y}\right)+J_{b} .
$$

Note that the second term on the right-hand side represents the $z$ component of $\operatorname{rot} B^{*}$. Since $B^{*}$ does not induce vacuum currents, $\operatorname{rot} B^{*}=0$, this term vanishes. Substituting Eqs. (1), (2), and (4) in Eq. (11), we obtain the desired modified sineGordon-type equation:

$$
\frac{\partial^{2} \phi}{\partial \tilde{x}^{2}}-\frac{\partial^{2} \phi}{\partial \tilde{t}^{2}}-\alpha \frac{\partial \phi}{\partial \tilde{t}}=\sin \left(\phi+\varphi^{*}\right)-j_{b}+\frac{\partial^{2} \varphi^{*}}{\partial \tilde{t}^{2}}+\alpha \frac{\partial \varphi^{*}}{\partial \tilde{t}} .
$$

Here, space, $\tilde{x}=x / \lambda_{J}$, is normalized by the Josephson penetration depth, $\lambda_{J}=\sqrt{\frac{c \Phi_{0}}{8 \pi^{2} d_{\text {eff }} J_{c 0}}}$ and time, $\tilde{t}=\omega_{p} t$, by the 
inverse plasma frequency $\omega_{p}^{-1}=\sqrt{\frac{\Phi_{0} C}{2 \pi c A J_{c 0}}}, \alpha=\left(\omega_{p} R_{n} C\right)^{-1}$ is the damping parameter and $j_{b}=J_{b} / J_{c 0}$. Equation (12) should be solved with respect to $\phi$ for the known $\varphi^{*}(x, t)$ with boundary conditions at the junction edges $x=0, L_{x}$ :

$$
\frac{\partial \phi}{\partial \tilde{x}}\left(0, L_{x}\right)=\frac{2 \pi d_{\mathrm{eff}} \lambda_{J}}{\Phi_{0}} H .
$$

Note that thanks to separation of variables, Eq. (8), the local nonuniform field drops out from the boundary conditions. This occurs because at the junction edges $B_{y}\left(0, L_{x}\right)=H+$ $B_{y}^{*}\left(0, L_{x}\right)$ so $B_{y}\left(0, L_{x}\right)-B_{y}^{*}\left(0, L_{x}\right)=H$, which together with Eq. (10) yields Eq. (13).

In what follows, we will normalize magnetic field by $H_{0}=$ $\Phi_{0} / \pi \Lambda_{1} \lambda_{J}$ and voltage by $V_{0}=\Phi_{0} \omega_{p} / 2 \pi c$, where $\Lambda_{1}=t_{i}+$ $\lambda_{1} \operatorname{coth}\left(d_{1} / \lambda_{1}\right)+\lambda_{2} \operatorname{coth}\left(d_{2} / \lambda_{2}\right), t_{i}$ is the junction interlayer width, $d_{1,2}$ are the widths, and $\lambda_{1,2}$ are the London penetration depths of the two electrodes (for details, see Ref. [36]).

\section{STATIC CASE}

In the static case, the only current component is the supercurrent $J_{s}$, Eq. (1), and Eq. (12) is reduced to

$$
\frac{\partial^{2} \phi}{\partial \tilde{x}^{2}}=\sin \left(\phi+\varphi^{*}\right)-j_{b}
$$

According to Eqs. (8)-(10), the local supercurrent density $J_{s}(x)$ directly depends on the local field $B^{*}(x)$. The experimentally measurable quantity, however, is the critical current $I_{c}$, which represents the maximum value of the integral of $J_{s}$ along the junction length. The value of $I_{c}$ alone does not disclose the $B^{*}(x)$ distribution. However, as we will show below, the $I_{c}(H)$ modulation pattern does carry information about distribution of the magnetic induction in the junction.

\section{A. Short junctions}

First, we consider the simplest case of a short junction $L_{x} \lesssim \lambda_{J}$. In this case, we may neglect magnetic field screening in the junction, i.e., set the second derivative term on the left-hand side of Eq. (14) to zero. From Eq. (13), we explicitly obtain $\phi(x) \simeq\left(2 \pi d_{\text {eff }} H / \Phi_{0}\right) x+\phi_{0}$, where $\phi_{0}$ is the integration constant. The total supercurrent is calculated directly by integration of $\sin \left[\phi(x)+\varphi^{*}(x)\right] d x$. The critical current is obtained by maximization with respect to the integration constant $\phi_{0}$.

To demonstrate how the local inhomogeneous field distorts the $I_{c}(H)$ pattern, we consider the case when the local field is created by stray fields from an Abrikosov vortex. This case has been described in detail in a recent work [28], in which it was shown that vortex-induced Josephson phase shift is well described by the equation

$$
\varphi^{*}(x)=-V \arctan \left(\frac{x-x_{v}}{\left|z_{v}\right|}\right),
$$

where $V$ is the vorticity ( +1 for a vortex, -1 for an antivortex), $x_{v}$ is the coordinate of the vortex along the junction and $z_{v}$ is the distance to the junction. Figure 2 shows (a) vortex stray fields, $-B^{*}(x)$, in the junction and (b) corresponding Josephson phase shifts $\varphi^{*}(x)$ for an Abrikosov vortex, $V=1$, at four different distances $z_{v}$ to the $\mathrm{JJ}$ along the junction

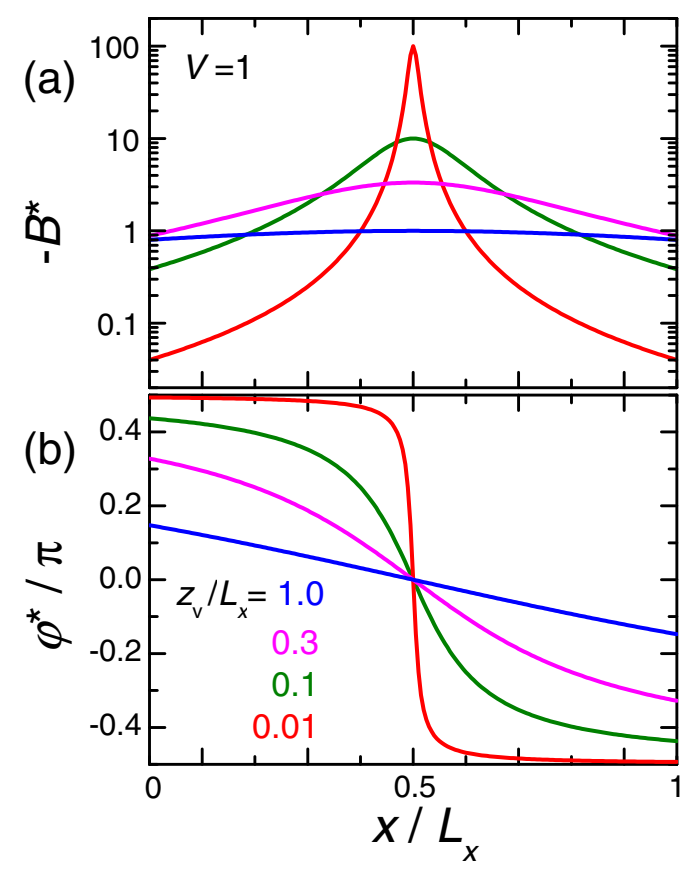

FIG. 2. (a) Abrikosov vortex-induced stray fields, $-B^{*}(x)$, at four different distances $z_{v}$ of the vortex to the junction and at $x_{v}=$ $0.5 L_{x}$ (in the semilogarithmic scale). The minus sign is due to the opposite sign of the vortex stray field with respect to the vortex. (b) Corresponding Josephson phase shifts $\varphi^{*}(x)$.

middle line $x_{v}=0.5 L_{x}$. The closer the vortex to the junction, the sharper and larger the local stray field $B^{*}(x)$. Note that the sign of the stray field is opposite to that in the vortex, leading to the minus sign in Eq. (15) [28].

Figure 3 illustrates an evolution of $I_{c}(H)$ patterns with changing local fields: row (a) upon approaching the vortex to the junction along the middle line $x_{v}=0.5 L_{x}$; row (b) upon moving the vortex from the left to the right side of the junction at $z_{v}=0.05 L_{x}$; and row (c) upon increasing the vorticity for a fixed position $x_{v}=0.5 L_{x}, z_{v}=0.05 L_{x}$. The quantity $\Theta_{v 1}$ represents the absolute value of the total vortex-induced Josephson phase shift $\Theta_{v 1}=\left|\varphi^{*}\left(L_{x}\right)-\varphi^{*}(0)\right|$, which yields the total local flux in the junction, $\left|\Phi^{*}\right|=\left(\Theta_{v 1} / 2 \pi\right) \Phi_{0}$.

The left panel in row (a) of Fig. 3 represents the case with far-away vortex, $z_{v}=100 L_{x}$, and negligible local field, $B^{*} \simeq 0$. In this case, $I_{c}(H)$ follows the standard Fraunhofer pattern. Figure 3(a) demonstrates that the $I_{c}(H)$ patterns get progressively distorted upon approaching the vortex to the JJ, accompanied by a subsequent sharpening and increasing of $B^{*}$, as shown in Fig. 2(a). Figure 3(b) corresponds to the case when only the position of $B^{*}$ maximum, $x_{v}$, changes, while the amplitude and the shape of $B^{*}$ remains the same. In Fig. 3(c), the position and the shape remain the same and only the amplitude of $B^{*}$ changes. Importantly, the distortion of $I_{c}(H)$ is individual and each pattern in Fig. 3 is clearly distinctive. As seen from Figs. 3(a)-3(c), the $I_{c}(H)$ patterns are sensitive to the shape, Figs. 2(a) and 3(a), the position, Fig. 3(b), and the amplitude, Fig. 3(c), of local field. Thus, the $I_{c}(H)$ contains encrypted information about local field distribution $B^{*}(x)$ and it should be possible to extract it by proper analysis. This supports the statement of 

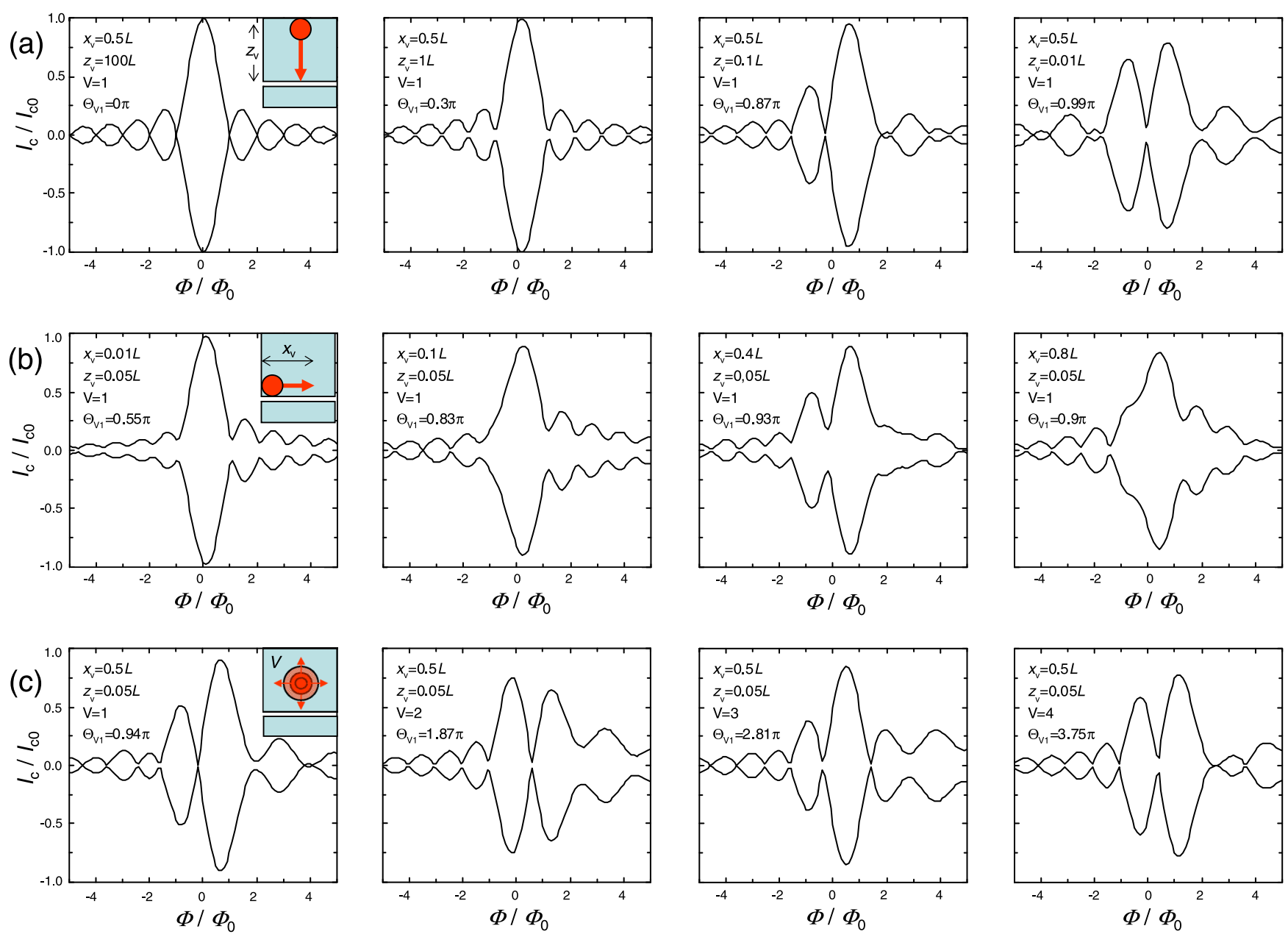

FIG. 3. Calculated $I_{c}(H)$ dependencies for a short junction with a static local field induced by stray fields from an Abrikosov vortex at different (a) distances, (b) positions, and (c) vorticities, as indicated in the insets. Panels in row (a) from left to right demonstrate a progressive distortion of $I_{c}(H)$ patterns upon the vortex approaching the junction along the middle line $x_{v}=0.5 L_{x}$. Panels in row (b): upon moving the vortex from the left to the right edge of the junction at constant distance $z_{v}=0.05 L_{x}$. Panels (c) show evolution of $I_{c}(H)$ patterns with changing the vorticity $V$ at a fixed vortex position $x_{v}=0.5 L_{v}, z_{v}=0.05 L_{x}$. Note that each $I_{c}(H)$ pattern is clearly distinguishable. This demonstrates that the effect of local inhomogeneous magnetic field is uniquely encoded in the shape of the $I_{c}(H)$ pattern. Therefore, it should be possible to reconstruct spatial distribution $B^{*}(x)$ from the analysis of $I_{c}(H)$ modulation.

Ref. [31] that spatial resolution of a scanning probe sensor based on a single planar junction is potentially not limited by its size. Such a device could obviate the trade-off problem between the field sensitivity and the spatial resolution inherent for scanning SQUID sensors [32,33], as mentioned in the Introduction.

\section{B. Long junctions}

For long JJs, $L_{x} \gg \lambda_{J}$, screening of magnetic field by the junction becomes significant. Simultaneously, JVs appear and start to affect junction properties. To obtain $I_{c}$ with static $B^{*}$, either an ordinary differential Eq. (14), or a dynamic partial differential Eq. (12) with time-independent $\varphi^{*}$ should be solved with boundary conditions, Eq. (13). Equation (14) is solved by a finite difference method with successive iterations and $I_{c}$ is determined as a maximum bias current at which a solution converges. Equation (12) is integrated explicitly using a central difference approximation and $I_{c}$ is determined using a threshold criterium for voltage. In case of a significant nonlinearity of $\varphi^{*}(x)$, the iterative solution of Eq. (14) may be quite sensitive to the initial approximation. On the other hand, the damping term in Eq. (12) allows less strict requirements to the initial approximation and usually provides faster convergence because, for the static case considered here, one can use a large $\alpha \simeq 1$ to speed up calculations. Therefore, all simulations shown below are obtained by solving partial differential Eq. (12).

Figure 4(a) shows a simulated $I_{c}(H)$ pattern for a long JJ with $L_{x}=10 \lambda_{J}$, in the absence of local field $B^{*}=0$. In contrast to the Fraunhofer $I_{c}(H)$ pattern for a short JJ, Fig. 3(a), it has a broad triangular central lobe, corresponding to the Meissner state [1]. Beyond it, JVs penetrate into the junction. Edge pinning of JVs, due to interaction with their own images [28], leads to metastability and multiply valued $I_{c}$. Some of the metastable states are seen in Fig. 4(a). To obtain those states, simulations are done by sweeping magnetic field back and forth in different field intervals. 

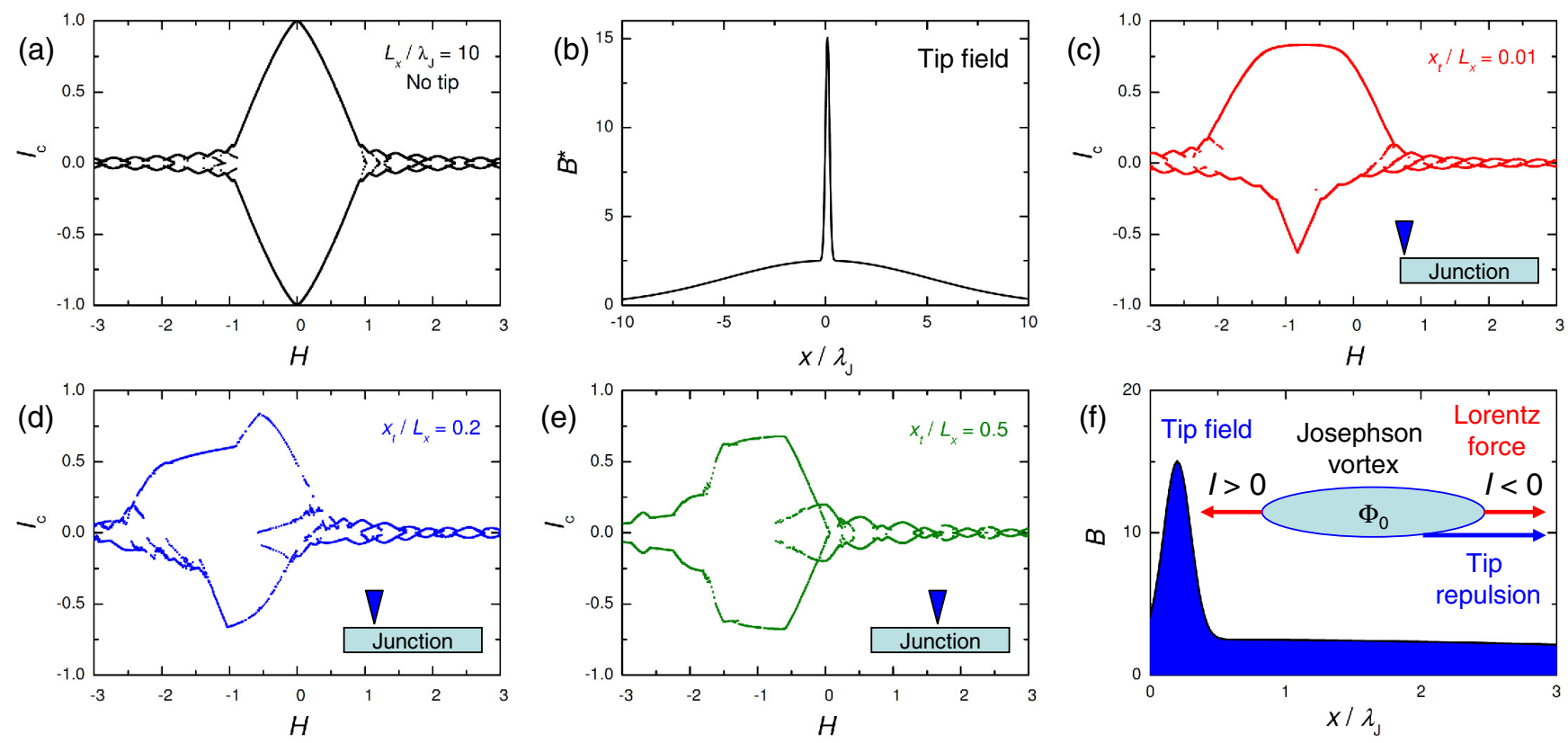

FIG. 4. Analysis of static characteristics of a long junction, $L_{x}=10 \lambda_{J}$, subjected to a local nonuniform field from a tip of magnetic force microscope. (a) $I_{c}(H)$ modulation without a tip. (b) The assumed profile of the tip field. (c)-(e) $I_{c}(H)$ modulation patterns for different location of the tip, $x_{t}$, as indicated in the insets. (f) A sketch of interaction of a Josephson vortex with the tip placed at the left edge of the junction $x_{t}=0.01 L_{x}$. Tip-induced local field and repulsion force are shown by the blue peak and arrow, respectively. Lorentz forces exerted by bias currents of different signs are depicted by red arrows. It is seen that the asymmetrically located local field creates a left-right asymmetry for JV motion and thus makes positive and negative critical currents dissimilar, as seen in panel (c).

Next we consider the case with a local field. Here we keep in mind another relevant case, when $B^{*}$ is induced by the MFM tip [29,30]. A standard MFM tip is covered by a thin ferromagnetic layer. Therefore, $B^{*}$ from the MFM sensor has a sharp dipole-type peak, originating from the end of the tip, and a broad background from the ferromagnetic layer at the cantilever. To mimic it, we approximate the tip-induced $B^{*}$ by two Gaussian peaks: a narrow one with the width $\Delta x_{1}=$ $0.1 \lambda_{J}$ containing a total flux of $0.5 \Phi_{0}$ and a broad $\Delta x_{2}=5 \lambda_{J}$ with the total flux $5 \Phi_{0}$, as shown in Fig. 4(b).

Figures 4(c)-4(e) show simulated $I_{c}(H)$ patterns for the same JJ with the MFM tip at different positions $x_{t}$ along the junction, as indicated in the insets. It is seen that the $I_{c}(H)$ in a long $\mathrm{JJ}$ is also distorted by the local field. However, there are certain differences with respect to the short junction case, Fig. 3.

\section{Asymmetry of $I_{c}(H)$ patterns}

From comparison of Figs. 3 and 4(c)-4(e), it can be seen that local fields lead to qualitatively different symmetries of $I_{c}(H)$ patterns for short and long JJs. In the absence of local field, $B^{*}=0$, the $I_{c}(H)$ patterns for both short and long JJs (with uniform parameters) are symmetric both with respect to field and current directions. That is, positive, $I_{c}^{+}$, and negative, $I_{c}^{-}$, critical currents are the same for positive and negative fields: $I_{c}^{+}(H)=-I_{c}^{-}(H), I_{c}^{+}(H)=I_{c}^{+}(-H)$, see Figs. 3(a) and 4(a).

Local field $B^{*} \neq 0$ removes the space symmetry of the problem. In all cases, this removes the symmetry with respect to field (space) inversion, $I_{c}(H) \neq I_{c}(-H)$. However, for short JJs, the symmetry with respect to current (time) inversion is preserved, $I_{c}^{+}(H)=-I_{c}^{-}(H)$. This occurs because, for short JJs, $I_{c}^{+}(H)$ and $I_{c}^{-}(H)$ correspond to maxima and minima of the same integral of $\sin \left[\left(2 \pi d_{\mathrm{eff}} H / \Phi_{0}\right) x+\varphi^{*}(x)+\phi_{0}\right] d x$. Those are achieved at some constant $\phi_{0}$ and $\phi_{0}+\pi$, respectively, thus leading to $I_{c}^{+}(H)=-I_{c}^{-}(H)$.

In long JJs, local field removes the current reversal symmetry as well, $I_{c}^{+}(H) \neq-I_{c}^{-}(H)$, see Figs. 4(c) and 4(d). This occurs because $I_{c}$ in long JJs has a different nature: It can be considered as a depinning current for JVs. Bias current exerts a Lorentz force on JVs and leads to the appearance of a fluxflow state with finite voltage. For the considered geometry, Fig. 1, positive $J_{b}$ pushes JVs to the left and negative- to the right, as indicated by red arrows in Fig. 4(f). In the absence of local field, $B^{*}=0$, JVs are pinned only at the edges of the junction due to attraction to image antivortices [28]. In this case, $I_{c}^{+}$and $I_{c}^{-}$correspond to depinning from the right and left edges, respectively, which are equal in the absence of physical nonuniformity of the JJ, as in Fig. 4(a).

The local inhomogeneous field with a finite gradient, $\partial B^{*} / \partial x \neq 0$, exerts an additional magnetic force on the $\mathrm{JV}$, as indicated by the blue arrow in Fig. 4(f), which will remove the symmetry between left and right edges and lead to $I_{c}^{+}(H) \neq$ $I_{c}^{-}(H)$, except for the case when the symmetric local field $B^{*}$ is placed symmetrically in the middle of the junction, as in Fig. 4(e). The local field also creates an additional pinning cite for JVs inside the JJ. This leads to a more profound metastability of $I_{c}(H)$ patterns as can be seen from comparison of Figs. 4(a) and 4(c)-4(e). 


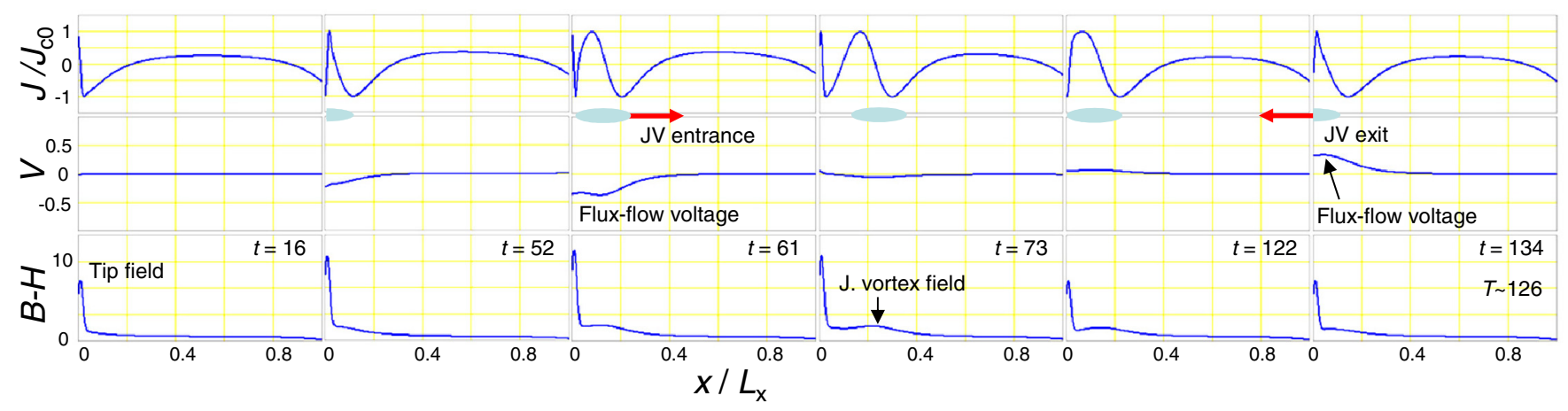

FIG. 5. Dynamics of a long junction $L_{x}=10 \lambda_{J}$ at $H=-0.55$ induced by an oscillating MFM tip placed at the left edge of the junction $x_{t}=0.01 L_{x}$. Six time frames are shown (time increasing from left to right) within approximately one period of tip oscillation. For each frame, the top panel shows a spatial distribution of the Josephson current, $\sin \left(\phi+\varphi^{*}\right)$, the middle panel—the voltage, $\partial\left(\phi+\varphi^{*}\right) / \partial t$, and the bottom panel-the inhomogeneous part of magnetic induction $B-H$. It is seen that the oscillating tip induces a shuttling motion of a single Josephson vortex, which enters and exits at the cite of the tip.

So far, we considered perfectly uniform JJs. However, real JJs often contain some nonuniformities, e.g., they may have spatial variation of intrinsic junction parameters, such as the critical current density, electrode thickness, bias current density, self-field effect, etc. In this case, the $I_{c}(H)$ pattern in the absence of local field must only be centrosymmetric, $I_{c}^{+}(H)=-I_{c}^{-}(-H)$ [5]. The latter is the consequence of space-time symmetry: simultaneous reversal of field (space) and current (time) is equivalent to flipping the junction upside down, which should not affect the output of the experiment. For nonuniform junctions, introduction of the local field $B^{*}$ removes all sorts of symmetry $I_{c}^{+}(H) \neq-I_{c}^{-}(H)$ and $I_{c}^{+}(H) \neq$ $I_{c}^{+}(-H)$ even for short junctions (not shown).

\section{DYNAMIC CASE}

The local field can be time-dependent, as, for example, in case of MFM in the tapping mode [30]. The timedependent local field $B^{*}(t)$ provides an additional driving force for junction dynamics, given by the last two terms on the right-hand side of Eq. (12). This can cause a flux-flow phenomenon induced by the oscillating MFM tip, as recently reported [30].

Figure 5 shows a time sequence of solutions of Eq. (12) with an oscillating MFM tip. Simulation parameters correspond to Fig. 4(c): $L_{x}=10 \lambda_{J}, x_{t} / L_{x}=0.01$ at $H=-0.55$. The damping parameter is $\alpha=0.5$. The top panels show spatial distributions of the Josephson current density, the middle panels - of voltage, and the bottom panels-magnetization $B-H$. We assume that the tip is oscillating harmonically, but the tip field is anharmonic due to the nonlinear distance dependence of the dipolelike tip field: $B^{*}(t)=B^{*}(0)[1+$ $a\left(1-\cos (\omega t)^{3}\right]$ with $a=0.5$ and $\omega=0.05 \omega_{p}$. Simulations are done for zero bias current $j_{b}=0$. Thus, all the dynamics is induced solely by the oscillating local field. Junction dynamics is periodic in time with the period of tip oscillations $T=2 \pi / \omega \simeq 125.7 \omega_{p}^{-1}$. Time sequences in Fig. 5 are shown for approximately one period of tip oscillations.

The considered field $H=-0.55$ corresponds to a large $I_{c}^{+}$ and a small $I_{c}^{-}$, see Fig. 4(c). As discussed above, see Fig. 4(f), for a long junction the asymmetry $I_{c}^{+}(H) \neq-I_{c}^{-}(H)$ makes left and right directions for motion of JVs inequivalent. This in turn can lead to a variety of unusual effects: The junction may act as a vortex-diode and rectify an external periodic or aperiodic signal [5]. For the case of a large $I_{c}^{+}$and a small $I_{c}^{-}$, the easy direction of JV motion is from left to right. From Fig. 5, it can be seen that with increasing the tip-induced field at $t \simeq 60 \simeq T / 2$, a Josephson vortex enters the junction from the left side, where the tip is placed. After that, it rapidly moves to the right, inducing a significant negative flux-flow voltage, see the middle panel at $t=61$. The JV penetrates to $x \sim 3 \lambda_{J}$, see the frame at $t=73$. As the tip retracts, the JV exits through the left edge, inducing a positive flux-flow voltage, see the frame at $t=134$. A slight delay between tip and vortex oscillations is caused by the viscosity of flux-flow motion due to a significant damping $\alpha=0.5$.

Figure 5 illustrates that the oscillating local field can induce a flux-flow phenomenon in the junction. Depending on parameters, it can be the shuttling in/out vortex motion, as in Fig. 5, or a more complex ratchetlike unidirectional motion with entrance of the JV from one side and exit from the other side of the junction. An example of such ratchetlike motion can be found in the Supplemental Material to Ref. [30]. In that case, every cycle, four JVs enter a junction from the left edge but only three leave from that side while one exits through the right edge, thus creating a ratchet effect, i.e., a net rectified unidirectional flux-flow motion induced by a periodic (or aperiodic) perturbation [5-8]. The back action of the tipinduced flux-flow motion leads to an additional damping of MFM tip oscillations, which can be detected in experiment. As discussed in Ref. [30], this provides a mechanism for detection of JVs by the MFM technique.

\section{CONCLUSIONS}

To conclude, we derived and numerically analyzed equations describing behavior of a JJ in local inhomogeneous magnetic field. As discussed in the Introduction, such a situation may have many different reasons and experimental realizations $[10-28,30]$. It was demonstrated that time-dependent local field creates an additional driving force, which may induce a flux-flow type dynamics in long junctions. This provides a mechanism for detection and manipulation of JVs by tapping-mode MFM [30]. The local inhomogeneous field 
removes the space-time symmetry of the junction and leads to a distortion of $I_{c}(H)$ modulation patterns. Importantly, the distortion uniquely depends on the spatial distribution of the local field $B^{*}(x)$ within the junction. Therefore, information about the local field profile is encoded into the shape of the $I_{c}(H)$ pattern and may in principle be reconstructed using an appropriate mathematical analysis. This strengthens an earlier argument that a single planar junction can be advantageously used as a scanning probe sensor [31]. The field sensitivity of such a sensor would depend on the area, similar to SQUID, but the spatial resolution would not be limited by the junction size. Therefore, a planar junction sensor can obviate the trade-off problem between the field sensitivity and the spatial resolution inherent in the scanning SQUID microscopy.

\section{ACKNOWLEDGMENTS}

I am grateful to T. Golod for assistance with assembling Fig. 3 and to V. V. Dremov, R. A. Hovhannisyan, S. Yu. Grebenchuk, and V. S. Stolyarov for stimulating discussions about MFM experiments. The work was supported by the Russian Science Foundation, Grant No. 19-19-00594. The paper was accomplished during a sabbatical semester at MIPT, supported by the 5 -top-100 program.
[1] A. Barone and C. Paterno, Physics and Applications of the Josephson Effect (J. Wiley \& Sons, New York, USA, 1982).

[2] S. A. Vasenko, K. K. Likharev, and V. K. Semenov, Static properties of distributed inhomogeneous Josephson junctions, Zh. Eksp. Teor. Phys. 4, 1444 (1981) [Sov. Phys. JETP 54, 766 (1981)].

[3] R. Fehrenbacher, V. B. Geshkenbein, and G. Blatter, Pinning phenomena and critical currents in disordered long Josephson junctions, Phys. Rev. B 45, 5450 (1992).

[4] A. Benabdallah, J. G. Caputo, and A. C. Scott, Exponentially tapered Josephson flux-flow oscillator, Phys. Rev. B 54, 16139 (1996).

[5] V. M. Krasnov, V. A. Oboznov, and N. F. Pedersen, Fluxon dynamics in long Josephson junctions in the presence of a temperature gradient or spatial nonuniformity, Phys. Rev. B 55, 14486 (1997).

[6] G. Carapella and G. Costabile, Ratchet Effect: Demonstration of a Relativistic Fluxon Diode, Phys. Rev. Lett. 87, 077002 (2001).

[7] H. B. Wang, B. Y. Zhu, C. Gürlich, M. Ruoff, S. Kim, T. Hatano, B. R. Zhao, Z. X. Zhao, E. Goldobin, D. Koelle, and R. Kleiner, Fast Josephson vortex ratchet made of intrinsic Josephson junctions in $\mathrm{Bi}_{2} \mathrm{Sr}_{2} \mathrm{CaCu}_{2} \mathrm{O}_{8}$, Phys. Rev. B 80, 224507 (2009).

[8] G. R. Berdiyorov, M. V. Milošević, L. Covaci, and F. M. Peeters, Rectification by an Imprinted Phase in a Josephson Junction, Phys. Rev. Lett. 107, 177008 (2011).

[9] G. R. Berdiyorov, M. V. Milošević, F. Kusmartsev, F. M. Peeters, and S. Savel'ev, Josephson vortex loops in nanostructured Josephson junctions, Sci. Rep. 8, 2733 (2018).

[10] D. J. Van Harlingen, Phase-sensitive tests of the symmetry of the pairing state in the high-temperature superconductors: Evidence for $d_{x^{2}-y^{2}}$ symmetry, Rev. Mod. Phys. 67, 515 (1995).

[11] C. C. Tsuei and J. R. Kirtley, Pairing symmetry in cuprate superconductors, Rev. Mod. Phys. 72, 969 (2000).

[12] H. Hilgenkamp, Ariando, H.-J. H. Smilde, D. H. A. Blank, G. Rijnders, H. Rogalla, J. R. Kirtley, and C. C. Tsuei, Ordering and manipulation of the magnetic moments in large-scale superconducting $\pi$-loop arrays, Nature 422, 50 (2003).

[13] A. Buzdin and A. E. Koshelev, Periodic alternating 0- and $\pi$-junction structures as realization of $\varphi$-Josephson junctions, Phys. Rev. B 67, 220504(R) (2003).

[14] J. Pfeiffer, M. Kemmler, D. Koelle, R. Kleiner, E. Goldobin, M. Weides, A. K. Feofanov, J. Lisenfeld, and A. V. Ustinov,
Static and dynamic properties of $0, \varphi$, and $0-\varphi$ ferromagnetic Josephson tunnel junctions, Phys. Rev. B 77, 214506 (2008).

[15] H. Sickinger, A. Lipman, M. Weides, R. G. Mints, H. Kohlstedt, D. Koelle, R. Kleiner, and E. Goldobin, Experimental Evidence of a $\varphi$ Josephson Junction, Phys. Rev. Lett. 109, 107002 (2012).

[16] S. V. Bakurskiy, N. V. Klenov, I. I. Soloviev, M. Yu. Kupriyanov, and A. A. Golubov, Superconducting phase domains for memory applications, Appl. Phys. Lett. 108, 042602 (2016).

[17] M. Weides, Magnetic anisotropy in ferromagnetic Josephson junctions, Appl. Phys. Lett. 93, 052502 (2008).

[18] C. Klose, T. S. Khaire, Y. Wang, W. P. Pratt, Jr., N. O. Birge, B. J. McMorran, T. P. Ginley, J. A. Borchers, B. J. Kirby, B. B. Maranville, and J. Unguris, Optimization of Spin-Triplet Supercurrent in Ferromagnetic Josephson Junctions, Phys. Rev. Lett. 108, 127002 (2012).

[19] A. Iovan and T. Golod, and V. M. Krasnov, Controllable generation of a spin-triplet supercurrent in a Josephson spin valve, Phys. Rev. B 90, 134514 (2014).

[20] I. A. Golovchanskiy, V. V. Bol'ginov, V. S. Stolyarov, N. N. Abramov, A. Ben Hamida, O. V. Emelyanova, B. S. Stolyarov, M. Yu. Kupriyanov, A. A. Golubov, and V. V. Ryazanov, Micromagnetic modeling of critical current oscillations in magnetic Josephson junctions, Phys. Rev. B 94, 214514 (2016).

[21] A. Iovan and V. M. Krasnov, Signatures of the spin-triplet current in a Josephson spin valve: A micromagnetic analysis, Phys. Rev. B 96, 014511 (2017).

[22] K. Lahabi, M. Amundsen, J. A. Ouassou, E. Beukers, M. Pleijster, J. Linder, P. Alkemade, and J. Aarts, Controlling supercurrents and their spatial distribution in ferromagnets, Nat. Commun. 8, 2056 (2017).

[23] T. Gaber, E. Goldobin, A. Sterck, R. Kleiner, D. Koelle, M. Siegel, and M. Neuhaus, Nonideal artificial phase discontinuity in long Josephson 0- $\kappa$ junctions, Phys. Rev. B 72, 054522 (2005).

[24] M. V. Miločsević, A. Kanda, S. Hatsumi, F. M. Peeters, and Y. Ootuka, Local Current Injection into Mesoscopic Superconductors for the Manipulation of Quantum States, Phys. Rev. Lett. 103, 217003 (2009).

[25] J. Sok and D. K. Finnemore, Thermal depinning of a single superconducting vortex in Nb, Phys. Rev. B 50, 12770 (1994).

[26] T. Golod, A. Rydh, and V. M. Krasnov, Detection of the Phase Shift from a Single Abrikosov Vortex, Phys. Rev. Lett. 104, 227003 (2010). 
[27] T. Golod, A. Iovan, and V. M. Krasnov, Single Abrikosov vortices as quantized information bits, Nat. Commun. 6, 8628 (2015).

[28] T. Golod, A. Pagliero, and V. M. Krasnov, Two mechanisms of Josephson phase shift generation by an Abrikosov vortex, Phys. Rev. B 100, 174511 (2019).

[29] H. Polshyn, T. R. Naibert, and R. Budakian, Manipulating multivortex states in superconducting structures, Nano Lett. 19, 5476 (2019).

[30] V. V. Dremov, S. Yu. Grebenchuk, A. G. Shishkin, D. S. Baranov, R. A. Hovhannisyan, O. V. Skryabina, I. A. Golovchanskiy, V. I. Chichkov, Ch. Brun, T. Cren, V. M. Krasnov, A. A. Golubov, D. Roditchev, and V. S. Stolyarov, Local Josephson vortex generation and manipulation with a magnetic force microscope, Nat. Commun. 10, 4009 (2019).

[31] T. Golod, O. M. Kapran, and V. M. Krasnov, Planar Superconductor-Ferromagnet-Superconductor Josephson Junctions as Scanning-Probe Sensors, Phys. Rev. Appl. 11, 014062 (2019).

[32] J. R. Kirtley, L. Paulius, A. J. Rosenberg, J. C. Palmstrom, C. M. Holland, E. M. Spanton, D. Schiessl, C. L. Jermain, J. Gibbons, Y.-K.-K. Fung, M. E. Huber, D. C. Ralph, M. B. Ketchen, G. W. Gibson, and K. A. Moler, Scanning SQUID susceptometers with sub-micron spatial resolution, Rev. Sci. Instrum. 87, 093702 (2016).

[33] A. Uri, A. Y. Meltzer, Y. Anahory, L. Embon, E. O. Lachman, D. Halbertal, N. HR, Y. Myasoedov, M. E. Huber, A. F. Young, and E. Zeldov, Electrically tunable multiterminal SQUID-onTip, Nano Lett. 16, 6910 (2016).

[34] A. A. Boris, A. Rydh, T. Golod, H. Motzkau, A. M. Klushin, and V. M. Krasnov, Evidence for Nonlocal Electrodynamics in Planar Josephson Junctions, Phys. Rev. Lett. 111, 117002 (2013).

[35] Equation (5) is correct for the uniform bias current distribution, $J_{b}(x)=$ const, in which case $B_{b}$ has only the $x$-component $B_{x b}$ in the junction. For a nonuniform $J_{b}(x), \operatorname{rot} B_{b}$ would also contain the $\partial B_{y b} / \partial x$ term. However, this term is usually negligible because the scale of variation in the $x$ direction is given by the large $\lambda_{J}$, while in the $y$ direction by the small electrode thickness $d \ll \lambda_{J}$. Therefore, $\left|\partial B_{x b} / \partial y\right| \gg\left|\partial B_{y b} / \partial x\right|$ and Eq. (5) remains a good approximation even for the nonuniform $J_{b}(x)$. Variation of $B_{b}$ at junction edges can also be neglected for a similar reason: Edge effects occur at the scale $\Delta x \sim d$, which for thin-film junctions is usually much smaller than the junction length $L_{x}$ even for short junctions $L_{x} \lesssim \lambda_{J}$.

[36] V. M. Krasnov, In-plane fluxon in layered superconductors with arbitrary number of layers, Phys. Rev. B 63, 064519 (2001). 